\title{
REVASCULARIZATION PROCEDURES FOR CHRONIC LOWER LIMB ISCHAEMIAIN TU TEACHING HOSPITAL
}

\author{
Bhomi K K*, Timala R B, Shrestha U K*, Koirala B*, Sharma G P*
}

\section{ABSTRACT}

A retrospective review of various revascularization procedures performed for the management of chronic lower limb ischaemia in Tribhuvan University Teaching Hospital (TUTH) during the last three and half years was done. A total of 37 revascularization procedures were done in 30 patients. Mean age of the patients was 53.10+15.34 (range 20-88) years. Except 3, all the patients were male. Atherosclerosis predominated as the cause of arterial occlusion. Superficial femoral artery was the commonest site of occlusion followed by external iliac artery. Femoropopliteal bypass was the commonest procedure done (62 per cent). Vascular prosthesis was used only in 7 patients, rest were managed using autogenous saphenous vein to bypass the occluded segment. Endarterectomy (10 per cent) and thrombectomy (8 per cent) were other procedures done for revascularization. There was $91 \%$ immediate limb salvage rate. Late limb salvage rate was $88 \%$ till the time of this writing.

Key Words: Revascularization, chronic leg ischaemia, bypass, graft.

\section{INTRODUCTION}

Vascular diseases are among the most common causes of morbidity and mortality in the western world. While coronary and carotid artery diseases are life threatening, peripheral vascular diseases are limb threatening and incur a substantial cost to the society.

Chronic lower limb ischaemia is due to progressive occlusion of the arteries supplying the lower limb. Atherosclerosis is the most common cause of this occlusion while these arteries are also afflicted by less common diseases, such as vasculitis, muscle entrapment syndromes, trauma and the fibro muscular disorders. Most of these patients are asymptomatic but a growing number of them are symptomatic with complaints ranging from mild claudication to gangrene of the limb. The increasing number of patients affected by this disease is, in part, due to the 'graying' of the general population and to the medical improvements of the past three decades that have allowed patients with generalized atherosclerosis to survive longer. ${ }^{1}$
Fortunately, diagnosis and management of chronic limb ischaemia have also significantly progressed in the past few decades resulting in increased limb salvage rates and improved quality of life for the patients.

\section{METHODOLOGY}

A retrospective study of all the patients undergoing surgical procedures for chronic lower limb ischaemia between September 1999 and March 2003 was done. Only those patients in whom surgical intervention was done to overcome arterial occlusion were included in this study. Patients who had undergone chemical lumbar sympathectomy as a sole procedure were excluded from the study though it was done in an attempt to improve limb perfusion. Excluded similarly were those who had undergone amputation of the limb without any attempt to overcome the arterial occlusion.

* T. U. Teaching Hospital, Maharajgunj, Kathmandu, Nepal.

Address for correspondence : Dr. Krishna Bhomi 
Selection criteria for revascularization procedure included incapacitating claudication and chronic critical leg ischaemia. Chronic critical leg ischaemia is defined on the basis provided by the Ad Hoc Committee on Reporting Standards, Society for Vascular Surgery and Second European Consensus document on critical leg ischaemia. ${ }^{2,3}$ Clinically this means patients with rest pain, ischemic ulcers or frank gangrene. Surgery was deferred in those who had functional ischaemia with mere mild to moderate claudication. All surgical candidates were subjected to arteriography of the involved limb. Surgery was performed under spinal anesthesia unless the aortoiliac segment is being addressed. Revascularization procedures were chosen depending on the site and nature of occlusion and the presence of suitable vein graft. Postoperative arteriogram was not a routine, unless graft occlusion was suspected.

Result was assessed during follow up by enquiring about relief of pain and resumption of functional activity. Objective assessment of improvement was done by clinical examination and with the use of hand held doppler.

Immediate limb salvage was defined as relief of ischemic pain

\section{Fig. 1}

Fig.1 : Left common iliac occlusion and right common iliac stenosis.

Fig. 2 and healing of ischemic lesion for 1 month after the first revascularization procedure. ${ }^{4}$ Further recording of limb status is being continued to calculate late limb salvage rate, which is defined as cumulative limb salvage rates for all patients having arterial reconstructive operation. ${ }^{4}$

\section{RESULTS}

During three and half year period, 30 patients underwent 37 revascularization procedures in 34 limbs. Only 3 patients (10 per cent) were female. Figure 4 shows age distribution of the patients. Mean age was $53.10+15.34$ (range 20-88) years. The youngest patient was 20 years old male who had occlusion of right superficial femoral artery. Femoropopliteal bypass with in situ saphenous vein graft was done for this patient and the leg ulcer, which was present for 6 months healed after the procedure. The oldest patient was 88 years old farmer with atherosclerotic occlusion of right superficial femoral artery and he also underwent femoropopliteal bypass with in situ saphenous vein.

Figure 5 shows presenting complaints of the patients. All the 30 patients had history of claudication at the time of presentation. Duration of claudication ranged from one month to 48 months with mean $15.5+15$ months. Eight patients (26.6 per cent) also had rest pain. Mean duration of rest pain was $5+9.32$ (range 1-24) months. One patient, 40 years old male presented with classical symptoms of the Leriche's syndrome with thigh and buttock claudication and impotence.

Smoking, diabetes mellitus, hypertension and hyperlipidemia are the major risk factors for peripheral arterial disease Number of patients having these risk factors is shown in fig. 6

Arteriography was done in all patients. Superficial femoral artery was the most common site of occlusion, followed by popliteal artery.

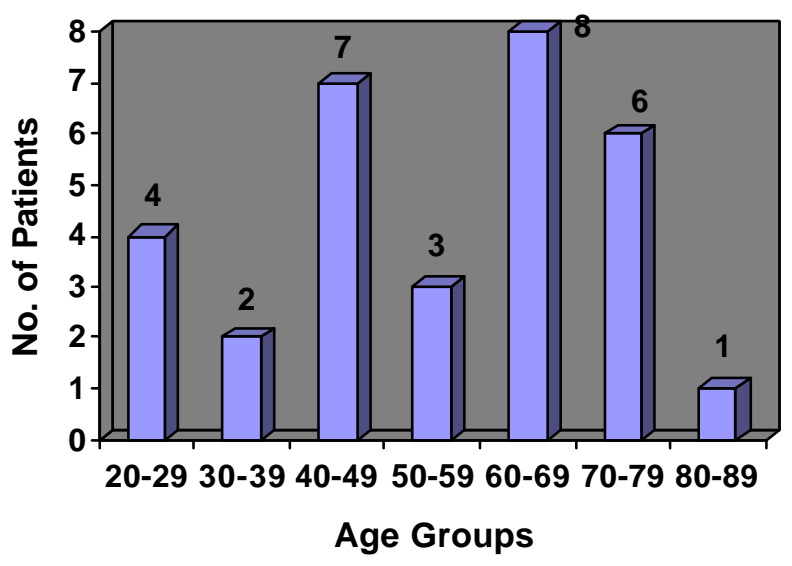

Fig.4 : Age Distribution of Patients 


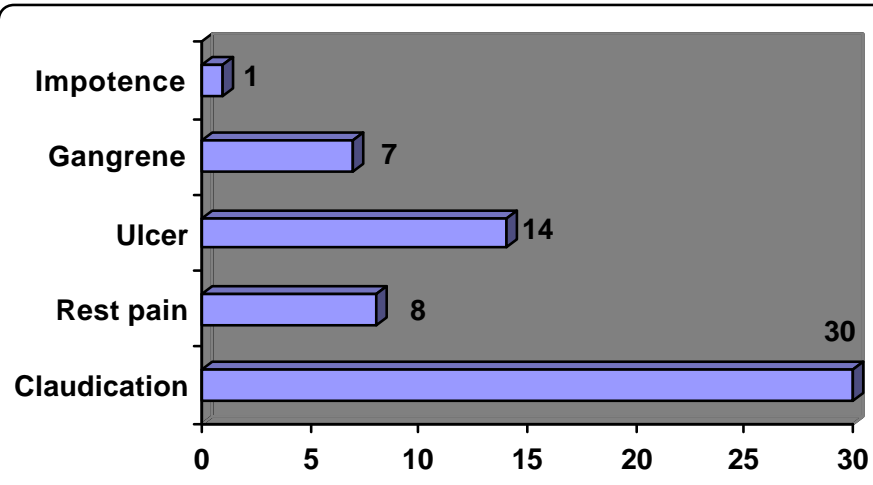

No. of Patients

Fig.5 : Presenting Complaints

Table I shows various procedures done for revascularization. Femoropopliteal bypass was the commonest procedure done $(n=23)$. Reverse saphenous vein graft was utilized in 19 patients. In situ saphenous vein and Polytetrafluoroethylene (PTFE) graft each were used in 2 patients.

Postoperatively, 3 patients needed re-operation for immediate postoperative graft thrombosis. All the patients had improvement in ischemic pain post operatively. Among the 14 limbs with ulcer, 11 had healing within 1 month. Immediate limb salvage rate thus was $91 \%$. None of the limbs needed amputation during the index admission.

One PTFE graft became infected and needed removal after 2 months. The same patient needed above knee amputation later on. Another patient who had undergone superficial femoral artery endarterectomy presented with repeated occlusion and below knee amputation had to be done after 4 months of initial procedure. Two other patients had persistent foot ulcer in spite of improved ischemic pain after revascularization. Late limb salvage rate calculated at the time of this writing therefore was $88 \%$.

Only 2 patients lost to follow up within 6 months after surgery. Till the time of this writing, 7 Patients lost to follow up; rest of the patients are reporting on the scheduled date. Mean duration of follow up was $26 \pm 14$ (range 4-46) months.

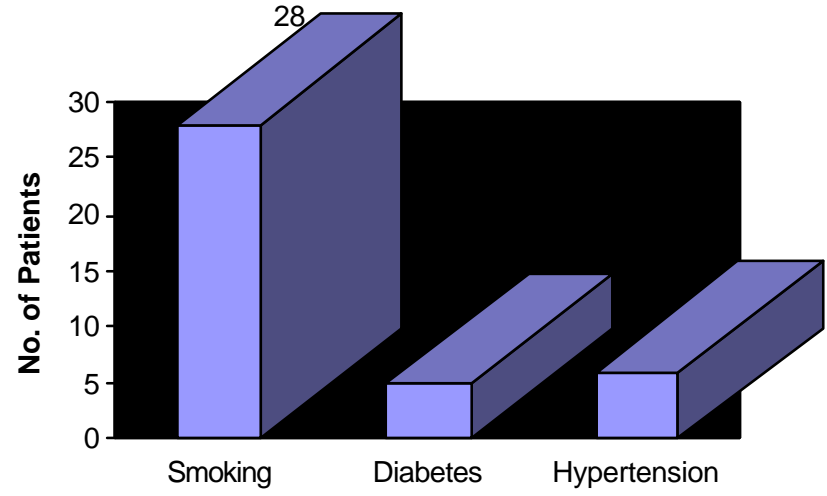

Fig.6 : Risk Factors for Peripheral Arterial Disease

\section{DISCUSSION}

A thorough understanding of the arterial obliterative process and its natural history is essential for the proper selection of candidates and appropriate surgical procedures to achieve limb revascularization. Atherosclerosis is the underlying cause of chronic limb ischaemia in the vast majority of patients. Majority of our patients had atherosclerotic disease of the involved arteries, though we did not have histopathological confirmation in every patient. The most important risk factors for atherosclerosis are hyperlipidemia, hypertension, cigarette smoking and diabetes mellitus. ${ }^{6,7}$ There was no complete data to incriminate hyperlipidemia as the risk factor, but 28 among 30 patients in this study were smokers and 86 per cent $(n=24)$ of them had cigarette index more than 500 .

Advancing age and male gender is associated with increased risk for atherosclerosis. Ninety per cent of our patients were male and majority of them were above 40 years of age, though we had a patient as young as 20 years of age. The arterial occlusion in the young patients is often associated with hyperlipidemia, hypercoagulable states, Buerger's disease or inborn errors of metabolism and progression of disease in these patients has been found to be especially rapid..$^{8,9,10}$ Because of lack of facilities, we did not look for hypercoagulability or metabolic disorders other than diabetes mellitus.

Table I : Procedures done for revascularization

\begin{tabular}{l|c}
\hline \multicolumn{1}{c|}{ Procedures } & Number \\
\hline Femoro popliteal bypass & 23 \\
\hline Femoro femoral bypass & 1 \\
\hline Endarterectomy & 4 \\
\hline Thrombectomy & 3 \\
\hline Ileofemoral bypass & 3 \\
\hline Aorto bifemoral inverse Y graft & 2 \\
\hline Excision with vein graft & 1 \\
\hline \multicolumn{1}{c}{ Total } & $\mathbf{3 7}$ \\
\hline
\end{tabular}


Our patients tend to present late in the course of the disease, probably because most of the patients are from hilly region, far away from the hospital. It may sound unbelievable that the mean duration of rest pain, which was present in 8 patients, was 5 months. Though all the patients had history of claudication, the most common indication of surgery was nonhealing ulcer of the foot or toe with or without rest pain

Conventional arteriography was done in all the patients selected for surgery. In addition to aiding in selection of candidates for bypass, it also displays the anatomic details required for the performance of an optimal surgical procedure. ${ }^{5}$

The most common site of atherosclerotic occlusion in the lower extremity is the transition between superficial femoral artery and the popliteal artery in the Hunter's canal. ${ }^{11}$ Other sites of predilection include the common iliac bifurcation and the mid popliteal artery. Eighteen among the 30 patients in the study had isolated occlusion of the superficial femoral artery. Five patients, all presenting late, had occlusion at two or more levels in the same arterial system and needed more than one procedure; two of them needed composite graft consisting of both synthetic and autogenous grafts.

A wide variety of procedures are now available to deal with chronic lower limb ischaemia. Bypass grafting has evolved as the suitable procedure in majority of the situations. Among the 37 revascularization procedures in our study, 30 were bypass grafting. Reversed autogenous saphenous vein has been the most consistently successful material. However, the resurgence of interest in using the saphenous vein in situ has resulted in a much greater utilization of the vein and may also improve the long-term patency. ${ }^{12}$ We utilized saphenous vein in all but 2 patients who underwent femoropopliteal bypass. In situ saphenous vein was utilized in only 2 patients because of unavailability of in situ valvulotome.

Watelet $\mathbf{J}$ et al carried out a randomized prospective study to compare in situ with reversed vein graft for femoropopliteal bypass. ${ }^{13}$ They found that limb salvage at 10 years was 73.5 per cent in the in situ graft group and 74.4 per cent in the reversed graft group. According to them, in addition to requiring a training period the in situ technique raises the problem of valve removal and the absence of these disadvantages makes the reversed technique the procedure of choice for femoropopliteal bypass. Further studies are necessary to draw any conclusion in our setup in this regard.

For larger caliber arteries like the abdominal aorta, there is no autogenous substitute available in the human body. Currently available synthetic grafts made of Dacron or PTFE offer a reasonable alternative with proven clinical efficacy. A prospective randomized trial comparing PTFE and vein graft indicated that they have nearly equal patency up to 2 years for femoro popliteal bypass. ${ }^{14}$ Thereafter, the rate of PTFE thrombosis is greater than that of vein; however, this difference in favor of vein grafts became statistically significant at 4 years only for popliteal bypasses below the knee. Whereas graft occlusion and infection are important clinical problems, the major limiting factor in our set up is their high cost. Among the 5 circumstances where synthetic grafts were used, one PTFE graft got infected and had to be removed.

The role of lumbar sympathectomy in the treatment of chronic lower limb ischaemia continues to be hotly debated. PerezBurkhardt JL et al did a retrospective study during a period of 5 years to know the current value of lumbar sympathectomy as isolated technique for the treatment of severe ischaemia of lower limbs in the absence of the possibility for vascular reconstruction. ${ }^{15}$ They evaluated 100 lumbar sympathectomy and concluded that lumbar sympathectomy could be a technique that moderately improves the ischemic limb where arterial reconstruction in not indicated. Advisability of adding lumbar sympathectomy to an arterial reconstruction remains controversial. ${ }^{5,16}$ Chemical lumbar sympathectomy was done in addition to revascularization procedure in 5 patients included in this study. These were done basically to buy time before definitive surgeries were performed.

Hemorrhage, thrombosis or occlusion of the graft and infection are common complications of bypass surgery. Hemorrhage occurs infrequently but requires immediate exploration. 5 Fortunately none of our patients had bleeding in the postoperative period.

The results of aggressive reoperation for failed bypasses have been surprisingly good. ${ }^{17}$ In a series of 318 femoropopliteal bypasses by Veith FJ et al, 13 (4 per cent) failed during the first month after operation and all were treated by reoperation. ${ }^{17}$ Three patients ( 8 per cent) in our study group had early thrombosis requiring re-exploration. One of them had a kink in the vein graft, while the cause could not be identified in the other two. However, graft function could be restored in all the three cases and we could achieve immediate limb salvage rate of $91 \%$, which compares well with $86 \%$ immediate limb salvage rate in a study by Veith FJ et al. ${ }^{4}$

Infection of the graft usually has devastating consequences. One patient in our study had infection of a PTFE femoropopliteal graft. The graft had to be removed and the patient landed with above knee amputation 2 months after the initial procedure. 
Veith FJ et al had late limb salvage rate of $66 \%$ in patients who survived 5 years after reconstructive procedure. ${ }^{4}$ It is too early to draw conclusion on late limb salvage rate in our study, though it is $88 \%$ till the time of this writing.

The reported operative mortality rates for infrainguinal bypass range between 0 and 7.5 per cent and the cause of death is most commonly complication of atherosclerosis of coronary or cerebral arteries. ${ }^{18,19,20}$ These low operative mortality contrasts with the high late death rates. A large study involving 551 patients undergoing lower limb revascularization for limb threatening ischaemia revealed $52 \%$ mortality within 5 years of the procedure. ${ }^{4}$ Fortunately there was no mortality during or in the early post-operative period in the study patients.

\section{CONCLUSION}

Surgical management of chronic lower limb ischaemia is being performed in TUTH with good results. Proper selection of candidates for operation and the appropriate surgical procedures are the keys to ensure the maximal improvement in limb perfusion with least risk to the patient. Further clinical studies with long term follow up is necessary to clarify the long term outcome of revascularized patients in Nepalese perspective.

\section{REFERENCES}

1 Sanchez LA Veith F. Dagnosis and treetnert of draric I over extrenity i schaenina. Vasc N®d 1998; 3(4): 291-9.

2 The Ad Hbc Comnittee on Peporting Standards, Soci ety for Vascul ar Surgery. Suggested standards for reports deal ing with I over extreninty i schaenna. J Vasc Surg 1986; 4: 80-94.

3 Second European Consensus Docunent on Chroni c Gri ti cal Leg I schaenna. European Vorkshop Group on cri ti cal I eg i schaenina. G rcul ati on 1991; 84(Supd 4): 1-26.

4 Veith 月, Guta SK Sanson PHet a. Progress in I i nb sal vage by reconst ructi ve arteri al surgery conbi ned with new or i nproved adj uncti ve procedures. Am Surg 1981; 194: $386-$ 400.

5 Robert B Rutherford. Vascul ar surgery $3^{\text {rd }}$ ed 1989; WB Sounders.

6 Rosen $A$, DePal ma RG Ri sk factors i n peri pheral atheroscl erosi s. Arch Surg 1973; 107: 303.
7. Cordon T, Karnd Pred sposi ti on to atherosd erosi s in the head, heart and I egs. The Franinghamstudy. J ANA 1972; 221: 661 .

8 DeBakey ME, Cravford ES, Garette E et al: Ced usi ve di sease of the lover extrentities in patients 16 to 37 years of age Am Surg 1964; 159: 873.

9 E drup-J orgenson J, F ari gan J P, Brace L \& $a$. hypercoagul ab e states and I over li ib i schaerna in young adul ts. J Vasc Surg 1989; 9: 334 41.

10. Pai rd ero PC, Joyce JW Ski mer R et al. Lover I i nb i scharia in young adl ts: Prognosti c i nd i cati ons. J Vesc Surg 1984; I: 459-64.

11. Ha navici H Paterns of arteriosd eratic lesi ans of the lover extrenity. Arch Surg 1957; 95: 918.

12. Leather $\mathrm{PP}$, Shah $\mathrm{DM}, \mathrm{Buchbi}$ nder Det al: Further experi ence with the saphenous vei $\mathrm{n}$ used in si tu for arterial bypass. AnJ Surg 1981; 142: 506.

13. Vittel ef J, Saupy P, Nenerd JF et al. Fenoropod i teal bypass: in si tu or reversed vein grafts? Ten-yer resul ts of a randbrized prospecti ve study. Am Vasc Surg 1997 Sep, 11(5) : 510-9.

14. Veith FJ, Gupta SK, Ascer E et al. Si x year prospecti ve nol ti center randonized conpari son of autol ogous saphenous ve $n$ and expanded pd ytetrofl uoroethl ene grafts in i ifrai ngi ind arteri al reconstructions. J Vasc Surg 1996; 3: 104

15. Perez-Brkhardt J L, Gorzal ez-Faj ardo JA Martin JF et al . Lunbar synpathect ony as i sol ated techni que for the treatnert of I over I i nb ctrori c ischaema. J Card ovesc Surg (Tori m) $1999 \mathrm{Feb}$; 40(1): 7-13.

16. Cutl er BS, Thonpson Je, $\mathrm{K}$ ei nsasser $L j$ et al. Autogenous saphenous vei $\mathrm{n}$ fenoropopd i teal bypass: Anal ysis of 208 cases. Surgery 1976; 79: 325.

17. Vei th FJ, Gupt a SK, Wengerter KR et al. Changi ng arteri oscl erctic di sease patterns and nanagenert strateg es in I over I i mb threateri ng i schaerina. Am Surg 1990, 272 40214.

18. Andros G Harris RW Sal les-Cerra $S$ et d. Armveins for ateria revesal ari zation of leg Ateri ograptic and dirical doservations. J Vasc Surg 1986; 4: 416.

19. Bernhard $\mathrm{W}$, Astnore CS, Evans VE et al. Bypass grafting to di stal arteries for li inb sal vage Surg Gnecd Costet 1972, 135: 219.

20. Deneese JA Rob CG Autogenous venous grafts ten years I ater. Surgery 1977; 82: $7 / 5$. 